\title{
Probing the Atomic Structures of Synthetic Monolayer and Bilayer Hexagonal Boron Nitride Using Electron Microscopy
}

\author{
Roland Yingjie Tay, Jinjun Lin ${ }^{1}$, Siu Hon Tsang ${ }^{2}$, Dougal G. McCulloch, Edwin Hang Tong Teo ${ }^{1,3, *}$ \\ School of Science, Physics, RMIT University, Melbourne 3001, Australia \\ ${ }^{1}$ School of Electrical and Electronic Engineering, Nanyang Technological University, Singapore 639798, Singapore \\ ${ }^{2}$ Temasek Laboratories@NTU, Singapore 639798, Singapore \\ ${ }^{3}$ School of Materials Science and Engineering, Nanyang Technological University, Singapore 639798, Singapore
}

*Correspondence to:

Teo EHT,

Tel: +65-6790- 6371

E-mail: HTTEO@ntu.edu.sg

Received December 1, 2016

Revised December 14, 2016

Accepted December 14, 2016
Monolayer hexagonal boron nitride (h-BN) is a phenomenal two-dimensional material; most of its physical properties rival those of graphene because of their structural similarities. This intriguing material has thus spurred scientists and researchers to develop novel synthetic methods to attain scalability for enabling its practical utilization. When probing the growth behaviors and structural characteristics of h-BN, the use of appropriate characterization techniques is important. In this review, we detail the use of scanning and transmission electron microscopies to investigate the atomic configurations of monolayer and bilayer h-BN grown via chemical vapor deposition. These advanced microscopy techniques have been demonstrated to provide intimate insights to the atomic structures of h-BN, which can be interpreted directly or indirectly using known growth mechanisms and existing theoretical calculations. This review provides a collective understanding of the structural characteristics and defects of synthetic h-BN films and facilitates a better perspective toward the development of new and improved synthesis techniques.

Key Words: Hexagonal boron nitride, Monolayer, Bilayer, Scanning electron microscopy, Transmission electron microscopy

\section{INTRODUCTION}

The methodologies used to synthesize and explore monolayer and few-layer two-dimensional (2D) hexagonal boron nitride (h-BN) crystals have fascinated researchers worldwide because of the rich physics of h-BN's growth mechanisms, structural characteristics, and unique and outstanding properties, which are critical for many next-generation 2D-based electronic and optical applications (Britnell et al., 2012; Dean et al., 2010; Watanabe et al., 2009). The large-scale fabrication of such films presents unprecedented opportunities for future mass manufacturing and commercialization similar to those of the Si-based semiconductor industry today. In recent years, numerous research groups have attempted to grow atomically thin h-BN films and have succeeded in obtaining large films of relatively good quality (Jang et al., 2016; Kim et al., 2015; Tay et al., 2016b). One of the most successful and highly utilized methods developed thus far is chemical vapor deposition (CVD) onto catalytic metal substrates (Shi et al., 2010; Song et al., 2010). Briefly, the growth is carried out at temperatures of greater than $1,000^{\circ} \mathrm{C}$ using $\mathrm{BN}$-containing precursors that are introduced either by sublimation of ammonia borane or by bubbling of liquid borazine (Kim et al., 2013b; Park et al., 2014). Metals with planar surfaces such as $\mathrm{Cu}$ not only serve as a catalyst for decomposition of the gaseous precursors, thereby enabling the deposition of atomically flat films, but also inhibit multilayer growth because of the relatively low solubility of B and $\mathrm{N}$, thereby leading to surface-mediated growth (Kim et al., 2012; Tay et al., 2014a). In a well-controlled growth, these films can be scaled to single-atom thickness while extending over large areas, thus enabling wafer-scale production (Han et al., 2014;

(a) This is an open-access article distributed under the terms of the Creative Commons Attribution Non-Commercial License (http://creativecommons.org/licenses/by-nc/4.0) which permits unrestricted noncommercial use, distribution, and reproduction in any medium, provided the original work is properly cited.

Copyrights @ 2016 by Korean Society of Microscopy 
Song et al., 2015; Wu et al., 2016).

Although the prospect of directly using these large films in various applications such as the fabrication of $2 \mathrm{D}$ heterostructure devices is enticing, they may not be truly reliable compared to directly exfoliated flakes from bulk crystals because the crystal structures contain imperfections, contaminants and damage-induced defects that arise from various growth conditions and post-growth processes. For example, the grain sizes of CVD-grown h-BN films are usually in the range from a few to 100 micrometers, which indicates a potentially high degree of polycrystallinity. Furthermore, unlike graphene, h-BN has a binary configuration and its lack of inversion symmetry results in more complex bonding structures with unfavorable homonuclear bonds (Gibb et al.,
2013; Li et al., 2015; Liu et al., 2012). The presence of such defects is known to alter the properties of h-BN films (Becton \& Wang, 2015; Li et al., 2015; Mortazavi et al., 2015). Hence, to fully exploit these scalable films and achieve better growth procedures, deep insights into their structural configuration, defects, and growth behaviors are critical. In this review article, we give an overview of the various geometric shapes, atomic configurations, grain orientations, stacking faults, and boundary defects observed in monolayer and bilayer CVDgrown h-BN films on $\mathrm{Cu}$ substrates. We provide detailed interpretations of the images obtained via scanning electron microscopy (SEM) and transmission electron microscopy (TEM) and explain the occurrences of the aforementioned characteristics as they relate to h-BN growth mechanisms.
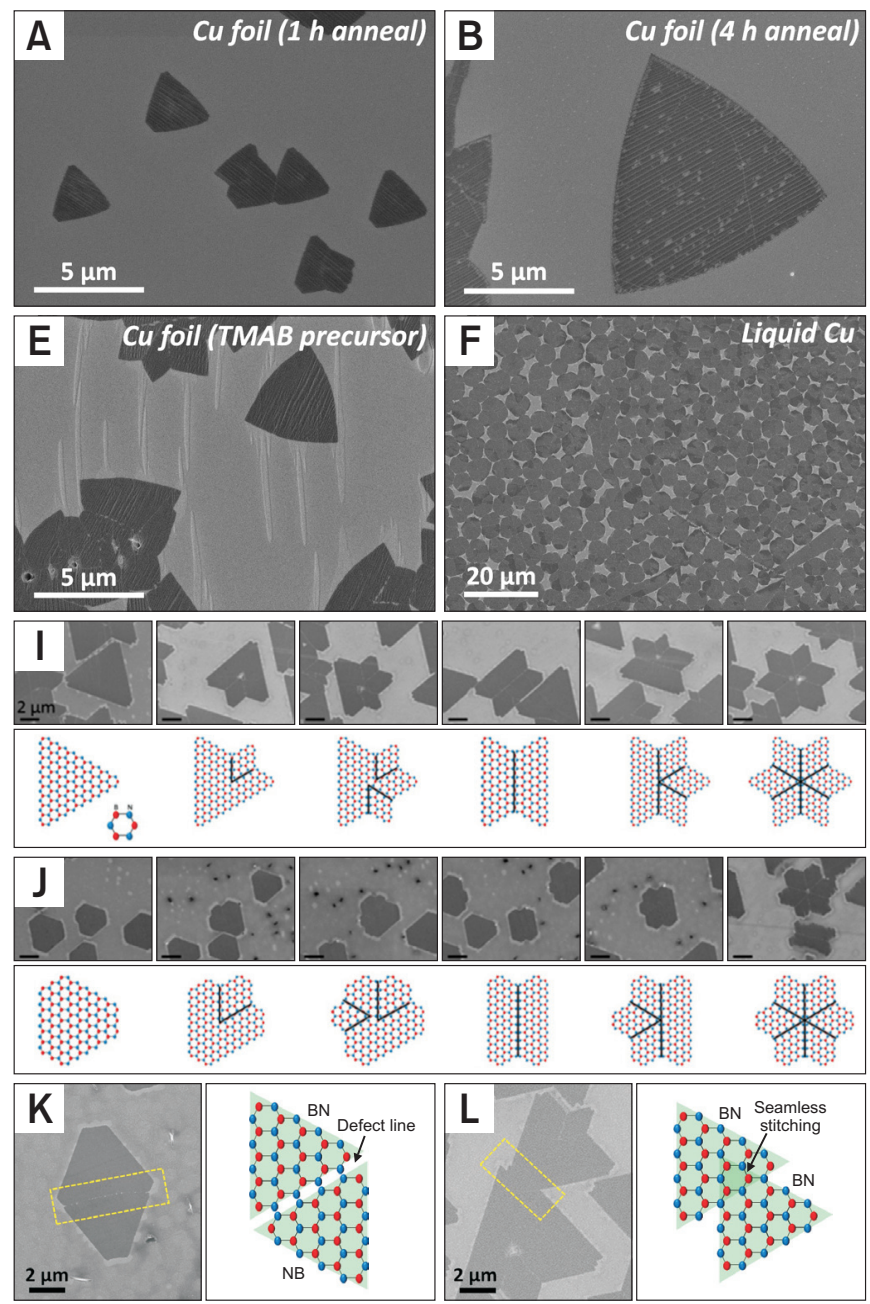
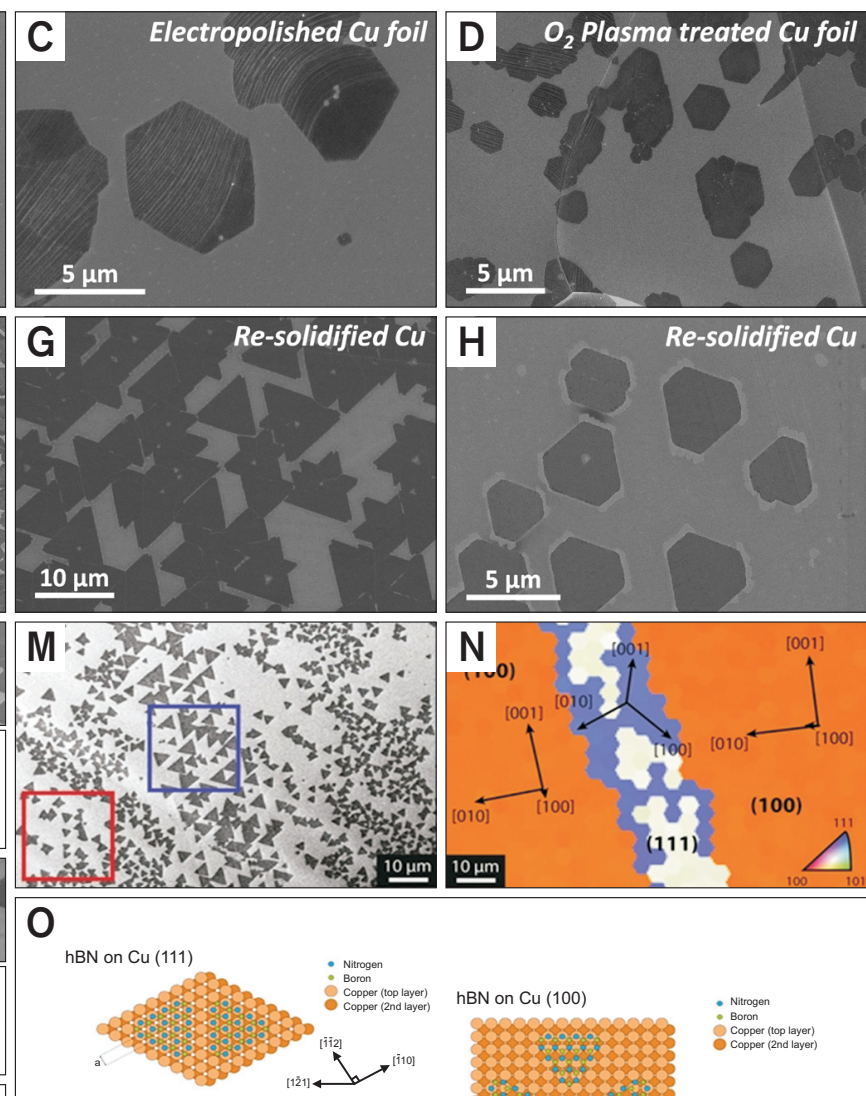

hBN on $\mathrm{Cu}(110)$
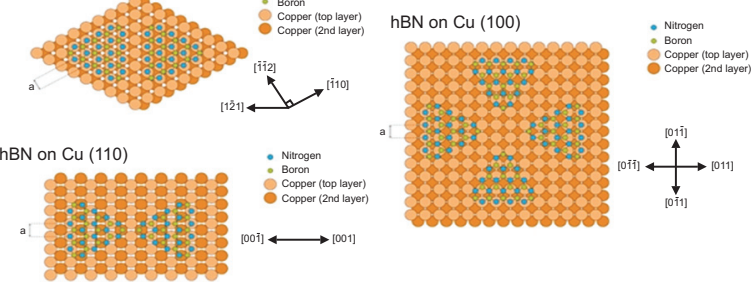

Fig. 1. Scanning electron microscopy (SEM) characterization of monolayer single-crystalline hexagonal boron nitride (h-BN) on Cu. (A-H) SEM images of h-BN domains grown under different growth conditions; the images depict their various geometric shapes and sizes. SEM images paired with their interpreted atomic configurations of the various multifaceted shapes formed by coalescence of multiple (I) triangular-shaped and (J) hexagonal-shaped domains. SEM image with its corresponding atomic configuration of (K) mirroring domains with a defect line and (L) aligned domains with a seamless boundary. (M) SEM image and its corresponding (N) electron backscatter diffraction (EBSD) map to correlate the h-BN orientations to the Cu facets. (O) Schematic of the atomic arrangement of h-BN on different Cu facets. Fig. 1I-K are reprinted from Tay et al. (2016b) (Nanoscale 8, 2434-2444) with the original copyright holder's permission. Fig. 1M-O are reprinted from Wood et al. (2015) (2D Mater. 2, 025003) with the original copyright holder's permission. 


\section{GEOMETRIC SHAPES AND EDGE ASSIGNMENTS}

SEM is a powerful tool for rapidly characterizing h-BN monolayers grown on $\mathrm{Cu}$ (or other metals) substrates and should be used directly after growth. Despite the h-BN layer being one atom thick, the h-BN domains can be identified as the regions with darker contrast with respect to the $\mathrm{Cu}$ substrate. Fig. 1A-H shows the SEM images of h-BN grown on various $\mathrm{Cu}$ substrates under different growth conditions (Tay et al., 2014a, 2014b, 2016a, 2016b). From these images, we can accurately account for the different shapes and sizes of the single-crystal domains. To date, the largest reported size of h-BN domains remains in the micrometer-scale regime (Lu et al., 2015). h-BN clearly tends to form regular shapes with well-defined edges, specifically, triangles and hexagons except for those grown on liquid $\mathrm{Cu}$ (Fig. 1F). Notably, some of the "irregular" shapes observed, such as that in the center of Fig. 1A, are formed by coalescence of neighboring domains; they are not representative of a single-crystalline structure. Interestingly, the circular domains grown on liquid $\mathrm{Cu}$ tend to form closely packed clusters (Fig. 1F). These circular shapes have been reported to be formed as a consequence of enhanced diffusion of $\mathrm{BN}$ radicals along the smooth flowing surface (Tan et al., 2015). Although the actual mechanism is not well understood, these domain shapes and their unique growth pattern are undoubtedly related to the growth kinetics differing from those of $\mathrm{h}-\mathrm{BN}$ grown on a solid surface.

In the case of the other typical domains with well-defined shapes, either triangles or hexagons, their edges are dictated by the different types of terminations. Ideally, three types of edges can exist: (i) zigzag edges with $\mathrm{N}$ terminations $\left(\mathrm{ZZ}_{\mathrm{N}}\right)$, (ii) zigzag edges with $\mathrm{B}$ terminations $\left(\mathrm{ZZ}_{\mathrm{B}}\right)$, and (iii) armchair edges with alternating $\mathrm{N}$ and $\mathrm{B}$ terminations. Theoretical calculations have revealed that $Z Z_{N}$ is the most energetically favorable edge (Liu et al., 2011); thus, if the domain shape is an equilateral triangle where the adjacent edges have an angle difference of $60^{\circ}$, then all of the edges should be assigned to $\mathrm{ZZ}_{\mathrm{N}}$. Indeed, most of the $\mathrm{h}-\mathrm{BN}$ domains that have been reported in the literature are dominated by triangular-shaped domains (Kidambi et al., 2014; Kim et al., 2012; Wang et al., 2014). However, for the formation of a nearly perfect hexagonal-shaped domain, such as those shown in Fig. 1C and $\mathrm{D}$, where the adjacent edges have an angle difference of $120^{\circ}$, the edges can either be assigned to alternating $Z Z_{N}$ and $\mathrm{ZZ}_{\mathrm{B}}$ or to purely armchair edges. Recent theoretical works have addressed this question and revealed that both $Z Z Z_{N}$ and $Z Z Z_{B}$ can coexist in a hexagonal structure when the chemical potential balance between $\mathrm{N}$ and $\mathrm{B}$ is appropriately tuned (Zhang et al., 2016; Zhao et al., 2015). Variations in the chemical potential of constituent elements can occur experimentally when different processes and substrates are used or when the Cu substrates are subjected to a pretreatment (i.e., oxidation) (Zhang et al., 2016), as in this scenario. To date, several other experimental approaches to obtaining similar hexagonal-shaped domains have been reported (Stehle et al., 2015; Tay et al., 2016b; Wang et al., 2015a; Wu et al., 2015; Yin et al., 2015b). The coexistence of $Z Z_{N}$ and $Z Z Z_{B}$ edges is further verified by the observation of truncated triangular-shaped domains (Fig. $1 \mathrm{H}$ ), where the long sides are assigned to $Z Z_{N}$ and the short sides to $Z Z_{B}$, consistent with the Wulff construction theory.

\section{SEAMLESS STITCHING AND DEFECT LINES}

Careful examination of the orientations of the h-BN domains in the SEM images reveals that they follow a strict epitaxial relation to the $\mathrm{Cu}$ lattices, as amplified in Fig. II and J. Multifaceted shapes such as "butterfly" and "6-apex star" consequently be observed when two or more adjacent domains coalesce in mirroring orientations (Tay et al., 2016b). Close inspection of the coalesced domains reveals defect lines, which are boundaries between two identical edge terminations that are unable to stitch because of unfavorable homonuclear bonding (N-N or B-B bonds) (Auwärter et al., 2003). Because $\mathrm{ZZ}_{\mathrm{N}}$ is assigned to all the edges for the triangular-shaped domains (and long sides for the truncated triangular domains), the interpreted atomic configurations of the h-BN domain(s) can hence be resolved, as shown below each SEM image.

Because $\mathrm{h}-\mathrm{BN}$ has a binary configuration, the lack of inversion symmetry where the unit cells are rotated by $60^{\circ}(\mathrm{BN}$ and $\mathrm{NB}$ ) results in opposite domain orientations. A classic example of the generation of a defect line is when two mirroring identical triangles merge to form a rhombus shape, as shown in Fig. $1 \mathrm{~K}$. Their $\mathrm{ZZ}_{\mathrm{N}}$ edges meet at the boundary and are unable to bond properly, thereby forming a defect line. Some of these linear defects split the coalesced domains symmetrically, whereas others are formed within the interior into a "lockand-key" structure (Wang et al., 2015b). Although the former defects, where both domains meet at their edges, are easy to visualize, the latter are slightly more complex. During the simultaneous growth of two mirroring domains, part of an edge of an h-BN domain is caved inward when an adjacent mirroring domain grows and extends toward it. The concave edge continues to recede to accommodate the growth front of the adjacent domain, finally resulting in the "lock-and-key" structure (Wang et al., 2015b). The occurrence of these defect lines is observed to be random, and they can coexist within various merged h-BN domains. However, when the h-BN domains are aligned (BN and $\mathrm{BN}$ ), they can seamlessly stitch at the boundary and preserve the single-crystalline structure as shown, in Fig. 1L (Wu et al., 2015; Yin et al., 2015a). Hence, the latter case is the key to obtaining a mosaic singlecrystalline film that can extend over large areas. 


\section{VAN DER WAALS EPITAXY}

To identify the epitaxial relation between $\mathrm{h}-\mathrm{BN}$ and $\mathrm{Cu}$, the crystallography of the $\mathrm{Cu}$ substrate can be mapped using electron backscatter diffraction (EBSD) and the mapped region can then be correlated to the orientations of the h-BN crystals using the aforementioned atomic configurations (Wood et al., 2015; Wu et al., 2015). In particular, for this analysis, triangular-shaped domains are preferred for the identification of a crystal's orientation. Fig. 1M shows a typical SEM image comprising sporadic triangular-shaped h-BN domains grown over several different $\mathrm{Cu}$ grains. The blue and red boxed regions show four and two different rotational orientations of the h-BN domains, respectively. Fig. $1 \mathrm{~N}$ shows the EBSD mapping image for the same region shown in Fig. 1M. Here the $\mathrm{Cu}$ surface orientation in the red box is identified as $\mathrm{Cu}(100)$, whereas that in the blue box is $\mathrm{Cu}$ (111). Fig. $1 \mathrm{O}$ shows the overlays of $\mathrm{h}-\mathrm{BN}$ domains on the various $\mathrm{Cu}$ surface facets. For a $\mathrm{Cu}(100)$ surface, the square lattice structure gives rise to four symmetrical rotations of h-BN configurations (rotated $90^{\circ}$ relative to one another), where the domain edge is parallel to $\mathrm{Cu}[011]$ or $\mathrm{Cu}[0 \overline{1} 1]$. By contrast, on the $\mathrm{Cu}$ (111) surface, their matching lattice constants $(\sim 1.8 \%$ lattice mismatch) and hexagonal symmetry allows for only two symmetrical rotations (rotated $180^{\circ}$ relative to each other), where the $\mathrm{N}$ and $\mathrm{B}$ atoms reside exclusively on both the hcp and fcc sites occupying the top and hollow sites of the metal, respectively (Laskowski et al., 2008). As previously mentioned, in both cases, the formation of mirroring pairs is because of the lack of inversion symmetry in BN and NB. To date, in addition to lattice-matching metals with high-index facets such as $\mathrm{Cu}$ and $\mathrm{Ni}$, aligned growths of $\mathrm{h}-\mathrm{BN}$ have been reported on $\mathrm{Ge}$ and $\mathrm{Al}_{2} \mathrm{O}_{3}$ substrates (Jang et al., 2016; Yin et al., 2015a).

\section{GRAIN ORIENTATIONS}

The h-BN grain orientations can be further determined by TEM with atomic precision inaccessible by SEM imaging. The directions of the h-BN lattices are identified by the orientations of the diffracted spots in their selected-area electron diffraction (SAED) pattern. After the sample is transferred onto a TEM grid, some of the single-crystalline domains are successfully preserved and their edges can be traced, as shown in Fig. 2A (Caneva et al., 2015). When multiple suspended regions in the grid holes within the domain are characterized by SAED, the single-crystalline nature is revealed by the identical positions of each diffracted
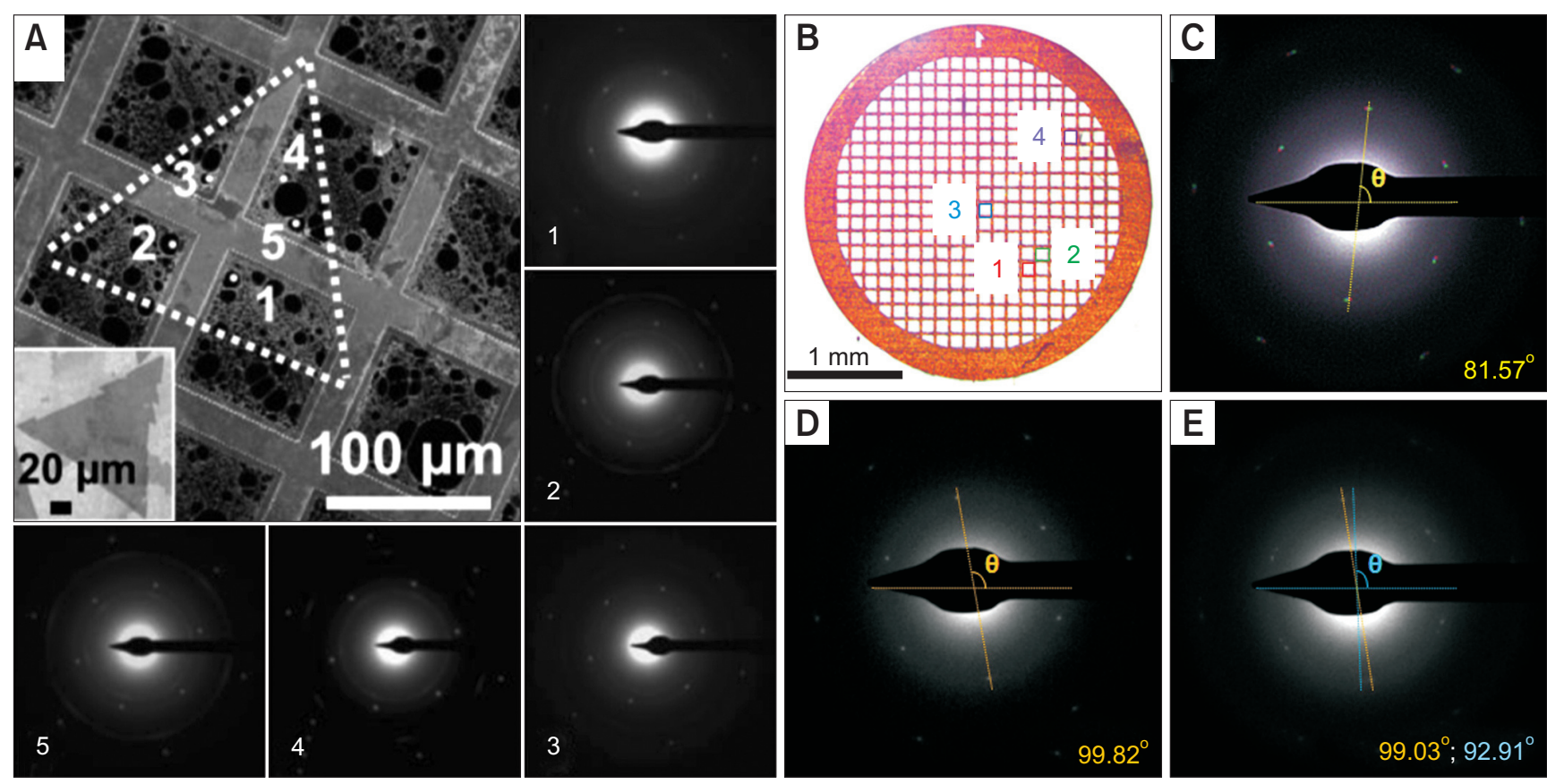

Fig. 2. Selected-area electron diffraction (SAED) mapping for the determination of grain orientation. (A) Transmission electron microscopy (TEM) image and SAED patterns acquired over various grid holes, as denoted 1 5, over a suspended triangular-shaped h-BN domain. (B) Photograph of a TEM grid after h-BN transfer. (C) Overlay of seven identical SAED patterns collected randomly over regions 1 4 in (B). (D) SAED pattern with a rotational mismatch relative to (C). (E) SAED pattern with two different orientations within a single grid hole. Fig. 2A is reprinted from Caneva et al. (2015) (Nano Lett. 15, 18671875) with the original copyright holder's permission. Fig. 2B-E are reprinted from Tay et al. (2016b) (Nanoscale 8, 2434-2444) with the original copyright holder's permission. 
spot (Fig. 2A). In addition, the diffraction spots that depict the lattice arrangements can be correlated to the traced edges that correspond to the $\mathrm{ZZ}$ directions, in agreement with the predicted theory of $Z_{\mathrm{N}}$ edges for triangular-shaped domains. The degree of polycrystallinity in a continuous monolayer h-BN film can also be determined using SAED. Fig. 2B shows a long-range mapping of over $1 \mathrm{~mm}$, where multiple SAED patterns were collected for various suspended h-BN films marked 1 4 on the TEM grid (Tay et al., 2016b). By measuring the angle of the straight line that connects two diffracted spots relative to a parallel line across the image where both intersect the center, the rotational difference in each SAED can be deduced. Fig. 2C shows an overlaid image of seven SAED patterns, indicating that the measured grains have an identical orientation; by contrast, Fig. 2D and E reveal grains with different orientations. Notably, SAED characterization cannot identify the inversion of the h-BN domains (BN and NB) because both will result in an identical

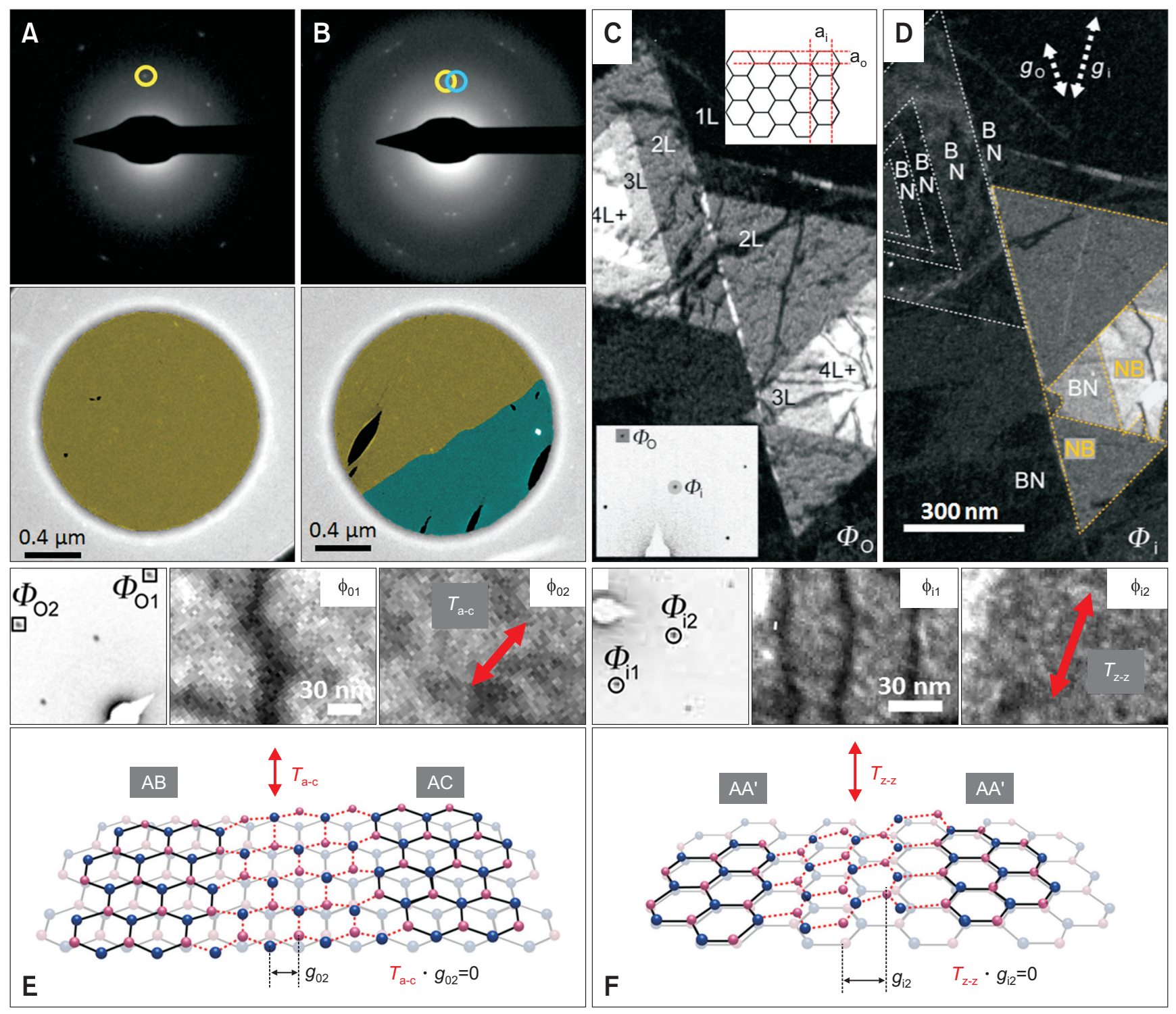

Fig. 3. Dark-field (DF)-transmission electron microscopy (TEM) characterization on monolayer and bilayer h-BN. Selected-area electron diffraction (SAED) patterns and their corresponding false-colored DF-TEM images of a (A) mono- and (B) bi-crystalline films, respectively. The yellow and blue regions in the DF-TEM images correspond to the orientations of the diffracted spots circled in yellow and blue, respectively. DF-TEM images of multilayer h-BN acquired by selecting (C) $\phi_{\mathrm{i}}$ and (D) $\phi_{\mathrm{o}}$ as shown in the bottom inset of (C). The top inset in (C) shows the d-spacing of the lattices, $a_{i}$ and $a_{o}$, corresponding to $\phi_{\mathrm{i}}$ and $\phi_{0}$, respectively. SAEDs with their corresponding DF-TEM images and schematics of strained regions for (E) AB/AC stacking and (F) AA' stacking by selecting $\phi_{01,02}$ and $\phi_{\mathrm{i} 1, i 2}$, respectively. Fig. 3A and B are reprinted from Tay et al. (2016b) (Nanoscale 8, 2434-2444) with the original copyright holder's permission. Fig. 3C-F are reprinted from Kim et al. (2013a) (Nano Lett. 13, 5660-5665) with the original copyright holder's permission. 
diffraction pattern. Hence, what appears as a single-crystalline film in Fig. 2C, may, in fact, comprise mirroring domains in a polycrystalline film. This possibility is important because mirroring domains generate defect lines which disrupt the pristine nature of a single crystal.

To physically identify the merged grains with different orientations, dark-field (DF) imaging is conducted for a selected diffraction spot while the other spots are blocked. The image appears bright for the grains in that specific orientation and dark for the others. Fig. 3A and B show the SAEDs and their corresponding false-colored DF-TEM images of mono- and bi-crystalline h-BN films, respectively (Tay et al., 2016b). For a single-crystalline film, no change in contrast is observed because electrons are diffracted in the same orientation throughout the film. However, for grains with misorientations, the lattices with different orientation(s) relative to the selected diffraction spot will be reflected as darker regions. Hence, mapping can be realized, and these images are used to quantify the grain sizes and precisely describe how the grains are merged.

\section{STACKING FAULTS AND TOPOLOGICAL DEFECTS}

Two stable stacking registries are well documented to exist for free-standing bilayer h-BN, specifically, $\mathrm{AA}^{\prime}$ and $\mathrm{AB} / \mathrm{AC}$ (Constantinescu et al., 2013). The stacking configurations are defined by two parameters: (i) the relative interlayer rotation angle $(\theta)$ and (ii) interlayer translation vector $(\tau)$ (Kim et al., 2013a). For AA' stacking, $\theta$ rotates by $60^{\circ}$ for the second layer relative to the first; by contrast, for $\mathrm{AB} / \mathrm{AC}$ stacking, $\tau$ shifts with no rotational difference. In both cases, heterogeneous pairs are overlapped where the energy is at minima. DF-TEM can also be used to identify the stacking configurations that exist in CVD-grown h-BN bilayers because the two different stacking configurations will result in either constructive or destructive interferences of the diffracted electrons (Kim et al., 2013a). Two sets of hexagonal diffraction spots ( $\phi_{i}$ and $\phi_{\mathrm{o}}$ ) indicate the lattice spacing of $a_{i}$ and $a_{o}$ (insets of Fig. $3 C)$, respectively. When imaging using $\phi_{0}$, no phase change occurs along $a_{o}$ for either type of stacking. Thus, the diffracted

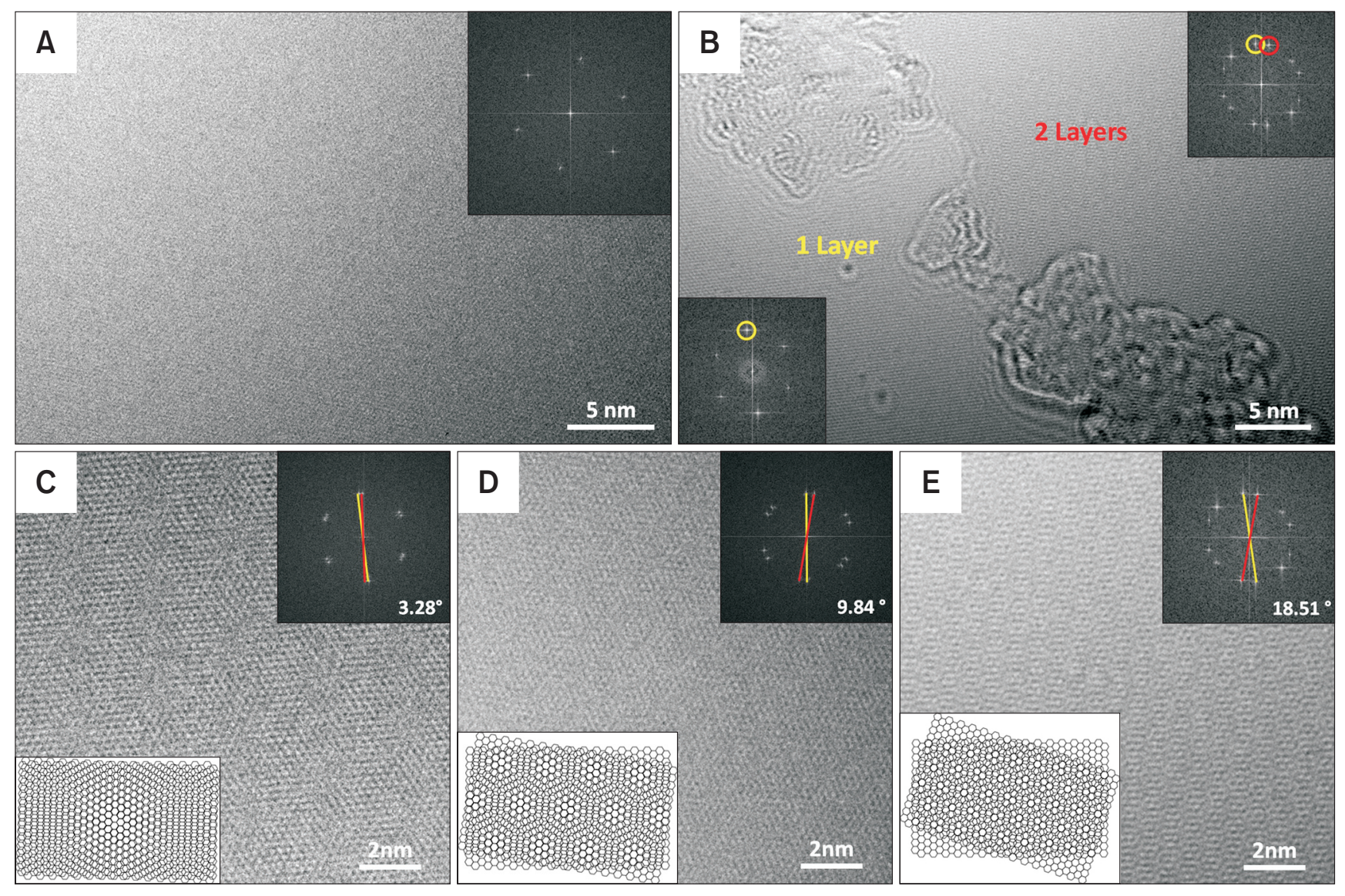

Fig. 4. Twisted bilayers and moiré patterns. High resolution-transmission electron microscopy (TEM) images of (A) a monolayer, (B) region with a second layer superimposed on the first, and bilayers with (C) $3.28^{\circ}$, (D) $9.84^{\circ}$, (E) $18.51^{\circ}$ rotational differences relative to the first layer. Top insets show the corresponding fast Fourier transforms. Bottom insets in (C-E) show the generation of moiré patterns with respect to their interlayer twist angle. 
electrons interfere constructively and the multilayer regions appear brighter than the monolayer regions (Fig. 3C). By contrast, when imaging using $\phi_{\mathrm{i}}$ (Fig. $3 \mathrm{D}$ ), in the case of AA' stacking, the heterogeneous pairs fully eclipse one another; thus, no phase difference is observed (i.e., constructive interference). In the case of $\mathrm{AB} / \mathrm{AC}$ stacking, however, phase shifts occur along $a_{i}$ by $2 \pi / 3$ and $4 \pi / 3$, which result in destructive interferences of diffracted electrons (Brown et al., 2012). Hence, by comparing the images in Fig. 3 C and D, the multilayer regions with $\mathrm{AA}^{\prime}$ or $\mathrm{AB} / \mathrm{AC}$ stacking can be determined, where the dark regions of the multilayers in Fig. $3 \mathrm{D}$ correspond to $\mathrm{AB} / \mathrm{AC}$ stacking.

Strain-induced topological defects are commonly observed on CVD-grown h-BN films (Kim et al., 2013a). When imaging using $\phi_{\mathrm{o}}$ and $\phi_{\mathrm{i}}$ for $\mathrm{AB} / \mathrm{AC}$ and $\mathrm{AA}^{\prime}$ stacking, respectively, as shown in Fig. $3 \mathrm{E}$ and F, nanometer-width dark lines are observed; however, these lines disappear when another diffraction spot is used (i.e., $\phi_{01}$ and $\phi_{02}, \phi_{\mathrm{i} 1}$ and $\phi_{\mathrm{i} 2}$ ). These dark lines can be interpreted as dislocations of the h-BN lattices along the first and second layer, where they are not properly aligned to their stable stacking registry. Only one of the diffracted spots will reflect the strained lattices because the lattice planes change for the particular orientation but remain aligned for the others. The topological defects are observed to always occur along the armchair direction for $\mathrm{AB} / \mathrm{AC}$ stacking bilayers and along the $\mathrm{ZZ}$ direction for $\mathrm{AA}^{\prime}$ stacking bilayers, as presented in the schematic illusions in Fig. $3 \mathrm{E}$ and F, respectively.

In addition to the intrinsically induced strains that occur during growth, synthetic h-BN films grown on metals are particularly vulnerable to damage during the subsequent transfer. High-resolution TEM images provide intimate details of the atomic configuration of h-BN films. In a nearly perfectly transferred monolayer film, the hexagonal lattice arrangement can be observed, as shown in Fig. 4A. However, in some other regions of the transferred film, folding can occur as a consequence of rough handling procedures. For example, in Fig. 4B, a second layer is superimposed over the first, with a different rotational orientation, as determined by fast Fourier transform analysis of the monolayer and bilayer regions. The relative twist in the second layer generates a moiré pattern. Fig. 4C-E show additional examples of the different moiré patterns formed by increasing the interlayer twist angle. The wavelength of the observed moire fringes decreases with increasing twist angle. Such unconventional stacking with interlayer twisting can change certain properties of the material, such as the magnetotransport properties of graphene (Schmidt et al., 2014).
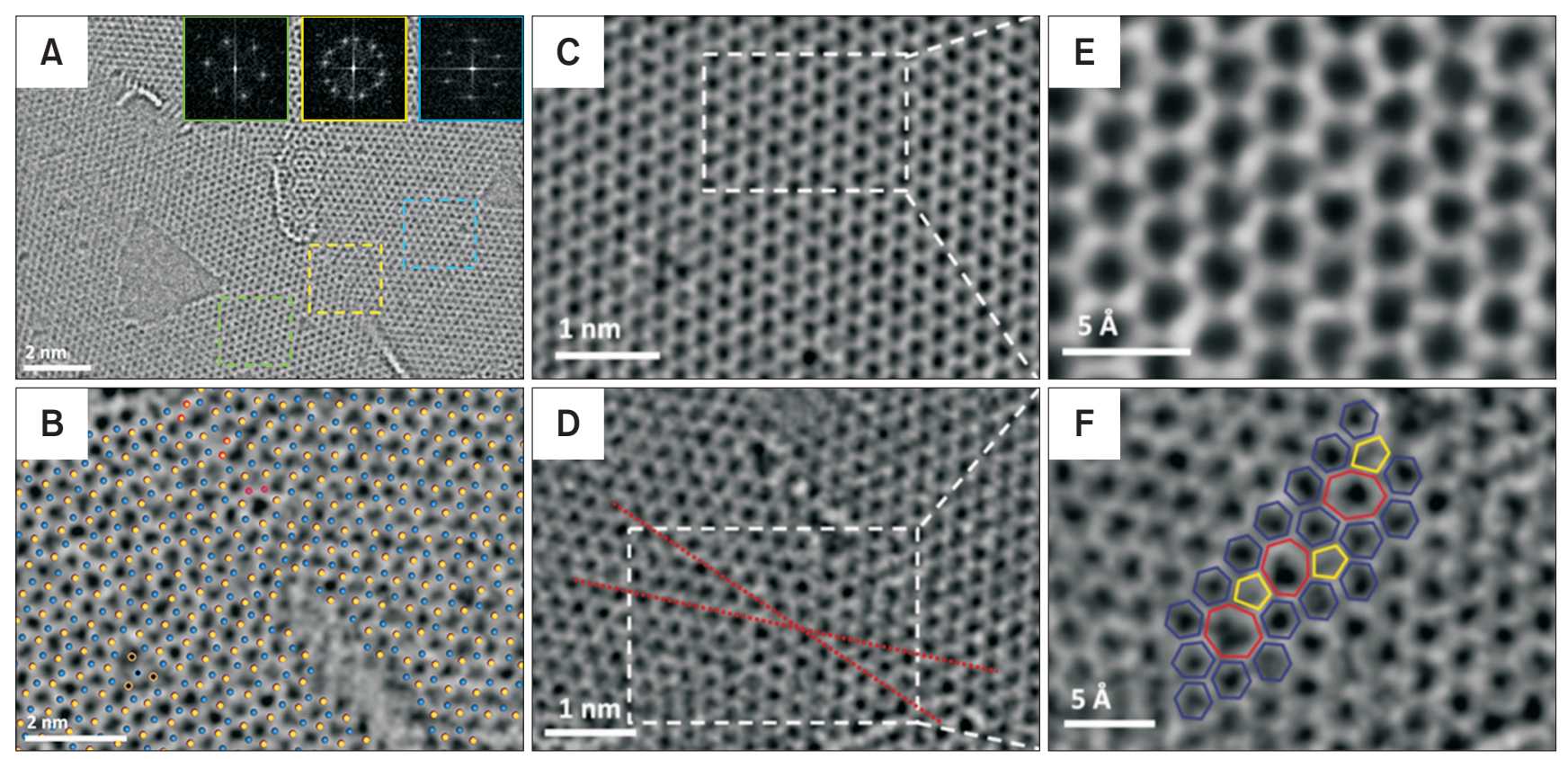

Fig. 5. Characterization of grain boundary defects. (A) Atomically resolved transmission electron microscopy (TEM) image of two merged grains with an orientation mismatch. Insets show the fast Fourier transforms taken over the respective boxed regions outlined in green, yellow, and blue. (B) Magnified image of the grain boundary in (A) with atomic assignments. Black spheres indicate vacancies, blue spheres indicate $\mathrm{N}$ atoms, yellow spheres indicate $\mathrm{B}$ atoms, and red circles indicate possible homonuclear bonds. TEM images of (C and E) a nearly perfect h-BN lattice structure and (D and F) along a grain boundary with the presence of $5 \mid 7$ pairs. Fig. 5A-F are reprinted from Gibb et al. (2013) (J. Am. Chem. Soc. 135, 6758-6761) with the original copyright holder's permission. 
Tay RY et al.

\section{GRAIN BOUNDARY DEFECTS}

Convergence of monolayer h-BN grains with rotational mismatch generates unfavorable atomic bonding structures along the boundaries, generally referred to as grain boundary defects; an example is shown in Fig. 5A (Gibb et al., 2013). The micrometer-sized polycrystalline grains produced by CVD would hence yield an abundance of such defects. To probe the types of bonding structures along these boundaries, atomically resolved TEM with a stringent set of parameters is required. Importantly, during TEM imaging, triangular-shaped vacancies induced by electron irradiation are commonly observed on h-BN films (Jin et al., 2009; Kotakoski et al., 2010; Park et al., 2015; Pham et al., 2016; Ryu et al., 2015). These holes are induced by monovacancies (B vacancies) formed upon electron-beam exposure. The formation of $\mathrm{B}$ vacancies instead of $\mathrm{N}$ vacancies is attributed to a lower knock-on energy threshold of B atoms $(79.5 \mathrm{keV})$ compared to that of $\mathrm{N}$ atoms $(118.6 \mathrm{keV})$ as well as to a lower B atomic displacement threshold energy $(19.36 \mathrm{eV})$ compared to that of $\mathrm{N}$ atoms $(23.06 \mathrm{eV})$ (Kotakoski et al., 2010). Both the $B$ and $N$ atomic displacement rates increase as defects transform from monovacancies into multivacancies, resulting in triangular shaped defects with $\mathrm{ZZ}_{\mathrm{N}}$ edges because the threshold energy of $\mathrm{N}$ displacement remained higher than B displacement (Kotakoski et al., 2010). Hence, a low acceleration voltage of $80 \mathrm{kV}$ (or lower) should be used and mapping of individual atoms can be accomplished by identifying the monovacancies as B atoms, as shown in Fig. 5B (Gibb et al., 2013).

Fig. 5C-F show atomically resolved TEM images of the crystalline structure within the interior of an $\mathrm{h}-\mathrm{BN}$ grain and along the grain boundary, along with their corresponding magnified images (Gibb et al., 2013). The images unambiguously reveal that the bonding structures along the grain boundary differ from the regular hexagonal structure comprising pentagon-heptagon pairs (5|7) (Gibb et al., 2013). In theory, and recently observed experimentally, another energetically more favorable structure exists; this structure is referred to as square-octagon pairs (4|8) (Liu et al., 2012; $\mathrm{Li}$ et al., 2015). The assigned atomic structures for the $5 \mid 7$ boundary include the presence of homonuclear B-B bonds, whereas the $4 \mid 8$ boundary solely comprises heteronuclear B-N bonds (Li et al., 2015). For both $5 \mid 7$ and $4 \mid 8$ boundaries, the band gap decreases in comparison to that of a perfect hexagonal structure, where the band gap of the $5 \mid 7$ structure is substantially lower than that of the $4 \mid 8$ structure ( $\mathrm{Li}$ et al., 2015). In view of the reduction in their band gaps, the existence of these grain boundaries may not be strictly detrimental because it could offer a way to tune the electronic properties of the h-BN films if appropriately engineered.

\section{EXPERIMENTAL METHODS}

Method details for images that are not reprinted can be found in the references cited in the main text. For Fig. 4, the images were collected by TEM (JEOL 2100F) operated at an acceleration voltage of $80 \mathrm{kV}$ and using an exposure time of $1 \mathrm{~s}$.

\section{CONCLUSIONS}

The geometric shapes and their edge assignments, epitaxial relation, stacking configurations, and various defects observed in synthetic monolayer and bilayer h-BN were analyzed and discussed in this review. Whereas the shapes and sizes as well as the method of growth for monolayers and bilayers may vary from one laboratory to the next, the mutual understanding of their atomic configurations remains unified. Characterization by SEM and TEM has been demonstrated to deliver invaluable insights into the atomic structures of h-BN, which can be interpreted directly or indirectly using known mechanisms or existing theoretical calculations. For characterizations that require h-BN films to be transferred onto other substrates such as TEM grids, the ability to distinguish between structural defects that originated intrinsically during growth and those caused by other subsequent post-growth processes such as transferring is critical. From a synthesis perspective, the quality of the films is determined by (i) grain sizes, (ii) grain orientations (including inversions), (iii) structural defects, and (iv) atomic smoothness. To achieve better quality films, all these aspects should be addressed. Indeed, the future commercialization and development of 2D-based electronics are highly dependent on the production of h-BN films because they are the best-known substrate for 2D materials to date.

\section{CONFLICT OF INTEREST}

No potential conflict of interest relevant to this article was reported.

\section{ACKNOWLEDGMENTS}

The authors acknowledge the funding support from the Australian Research Council (ARC) discovery project (DP130103003), NTU-A ${ }^{\star}$ STAR Silicon Technologies Centre of Excellence under the program grant No. 1123510003, and Singapore Ministry of Education Academic Research Fund Tier 2 No. MOE2013-T2-2-050. R.Y.T. gratefully acknowledges the facilities and the scientific and technical assistance of the RMIT Microscopy \& Microanalysis Facility at RMIT University. 
Auwärter W, Muntwiler M, Osterwalder J, and Greber T (2003) Defect lines and two-domain structure of hexagonal boron nitride films on $\mathrm{Ni}(111)$. Surf. Sci. 545, 735-740.

Becton M and Wang X (2015) Grain-size dependence of mechanical properties in polycrystalline boron-nitride: a computational study. Phys. Chem. Chem. Phys. 17, 21894-21901.

Britnell L, Gorbachev R V, Jalil R, Belle B D, Schedin F, Mishchenko A, Georgiou T, Katsnelson M I, Eaves L, Morozov S V, Peres N M R, Leist J, Geim A K, Novoselov K S, and Ponomarenko L A (2012) Field-effect tunneling transistor based on vertical graphene heterostructures. Science 335, 947-950.

Brown L, Hovden R, Huang P, Wojcik M, Muller D A, and Park J (2012) Twinning and twisting of tri- and bilayer Graphene. Nano Lett. 12, 1609-1615.

Caneva S, Weatherup R S, Bayer B C, Brennan B, Spencer S J, Mingard K, Cabrero-Vilatela A, Baehtz C, Pollard A J, and Hofmann S (2015) Nucleation control for large, single crystalline domains of monolayer hexagonal boron nitride via Si-doped Fe catalysts. Nano Lett. 15, 1867-1875.

Constantinescu G, Kuc A, and Heine T (2013) Stacking in bulk and bilayer hexagonal boron nitride. Phys. Rev. Lett. 111, 036104.

Dean C R, Young A F, Meric I, Lee C, Wang L, Sorgenfrei S, Watanabe K, Taniguchi T, Kim P, Shepard K L, and Hone J (2010) Boron nitride substrates for high-quality graphene electronics. Nat. Nanotechnol. $\mathbf{5}$ 722-726.

Gibb A L, Alem N, Chen J H, Erickson K J, Ciston J, Gautam A, Linck M, and Zettl A (2013) Atomic resolution imaging of grain boundary sefects in monolayer chemical vapor deposition-grown hexagonal boron nitride. J. Am. Chem. Soc. 135, 6758-6761.

Han J, Lee J Y, Kwon H, and Yeo J S (2014) Synthesis of waferscale hexagonal boron nitride monolayers free of aminoborane nanoparticles by chemical vapor deposition. Nanotechnology 25 145604.

Jang A R, Hong S, Hyun C, Yoon S I, Kim G, Jeong H Y, Shin T J, Park S O, Wong K, Kwak S K, Park N, Yu K, Choi E, Mishchenko A, Withers F, Novoselov K S, Lim H, and Shin H S (2016) Wafer-scale and wrinklefree epitaxial growth of single-orientated multilayer hexagonal boron nitride on sapphire. Nano Lett. 16, 3360-3366.

Jin C, Lin F, Suenaga K, and lijima S (2009) Fabrication of a freestanding boron nitride single layer and its defect assignments. Phys. Rev. Lett. 102, 195505.

Kidambi P R, Blume R, Kling J, Wagner J B, Baehtz C, Weatherup R S, Schloegl R, Bayer B C, and Hofmann S (2014) In situ observations during chemical vapor deposition of hexagonal boron nitride on polycrystalline copper. Chem. Mater. 26, 6380-6392.

Kim C J, Brown L, Graham M W, Hovden R, Havener R W, McEuen P L, Muller D A, and Park J (2013a) Stacking order dependent second harmonic generation and topological defects in h-BN bilayers. Nano Lett. 13, 5660-5665.

Kim G, Jang A R, Jeong H Y, Lee Z, Kang D J, and Shin H S (2013b) Growth of high-crystalline, single-layer hexagonal boron nitride on recyclable platinum foil. Nano Lett. 13, 1834-1839.

Kim K K, Hsu A, Jia X, Kim S M, Shi Y, Hofmann M, Nezich D, RodriguezNieva J F, Dresselhaus M S, Palacios T, and Kong J (2012) Synthesis of monolayer hexagonal boron nitride on $\mathrm{Cu}$ foil using chemical vapor deposition. Nano Lett. 12, 161-166.

Kim S M, Hsu A, Park M H, Chae S H, Yun S J, Lee J S, Cho D H, Fang W, Lee C, Palacios T, Dresselhaus M, Kim K K, Lee Y H, and Kong J (2015) Synthesis of large-area multilayer hexagonal boron nitride for high material performance. Nat. Commun. 6, 8862.

Kotakoski J, Jin C H, Lehtinen O, Suenaga $\mathrm{K}$, and Krasheninnikov $\mathrm{A}$ $\checkmark$ (2010) Electron knock-on damage in hexagonal boron nitride monolayers. Phys. Rev. B 82, 113404.

Laskowski R, Blaha P, and Schwarz K (2008) Bonding of hexagonal BN to transition metal surfaces: an ab initiodensity-functional theory study. Phys. Rev. B 78, 045409.

Li Q, Zou X, Liu M, Sun J, Gao Y, Qi Y, Zhou X, Yakobson B I, Zhang Y, and Liu Z (2015) Grain boundary structures and electronic properties of hexagonal boron nitride on $\mathrm{Cu}(111)$. Nano Lett. 15, 5804-5810.

Liu Y, Bhowmick S, and Yakobson B I (2011) BN white graphene with "colorful" edges: the energies and morphology. Nano Lett. 11, 31133116.

Liu Y, Zou X, and Yakobson B I (2012) Dislocations and grain boundaries in two-dimensional boron nitride. ACS Nano 6, 7053-7058.

Lu G, Wu T, Yuan Q, Wang H, Wang H, Ding F, Xie X, and Jiang M (2015) Synthesis of large single-crystal hexagonal boron nitride grains on Cu-Ni alloy. Nat. Commun. 6, 6160.

Mortazavi B, Pereira L F C, Jiang J W, and Rabczuk T (2015) Modelling heat conduction in polycrystalline hexagonal boron-nitride films. Sci. Rep. 5, 13228.

Park H J, Ryu G H, and Lee Z (2015) Hole defects on two-dimensional materials formed by electron beam irradiation: toward nanopore devices. Appl. Microsc. 45, 107-114.

Park J H, Park J C, Yun S J, Kim H, Luong D H, Kim S M, Choi S H, Yang W, Kong J, Kim K K, and Lee Y H (2014) Large-area monolayer hexagonal boron nitride on Pt foil." ACS Nano, 8, 8520-8528.

Pham T, Gibb A L, Li Z, Gilbert S M, Song C, Louie S G, and Zettl A (2016) Formation and dynamics of electron-irradiation-induced defects in hexagonal boron nitride at elevated temperatures. Nano Lett. 16, 7142-7147.

Ryu G H, Park H J, Ryou J, Park J, Lee J, Kim G, Shin H S, Bielawski C W, Ruoff R S, Hong S, and Lee Z (2015) Atomic-scale dynamics of triangular hole growth in monolayer hexagonal boron nitride under electron irradiation. Nanoscale 7, 10600-10605.

Schmidt H, Rode J C, Smirnov D, and Haug R J (2014) Superlattice structures in twisted bilayers of folded graphene. Nat. Commun. 5, 5742.

Shi Y, Hamsen C, Jia X, Kim K K, Reina A, Hofmann M, Hsu A L, Zhang K, Li H, Juang Z Y, Dresselhaus M S, Li L J, and Kong J (2010) Synthesis of few-layer hexagonal boron nitride thin film by chemical vapor deposition. Nano Lett. 10, 4134-4139.

Song L, Ci L, Lu H, Sorokin P B, Jin C, Ni J, Kvashnin A G, Kvashnin D G, Lou J, Yakobson B I, and Ajayan P M (2010) Large scale growth and characterization of atomic hexagonal boron nitride layers. Nano Lett. 10, 3209-3215.

Song X, Gao J, Nie Y, Gao T, Sun J, Ma D, Li Q, Chen Y, Jin C, Bachmatiuk A, Rümmeli, M, Ding F, Zhang Y, and Liu Z (2015) Chemical vapor deposition growth of large-scale hexagonal boron nitride with controllable orientation. Nano Res. 8, 3164-3176. 
Stehle Y, Meyer H M, Unocic R R, Kidder M, Polizos G, Datskos P G, Jackson R, Smirnov S N, and Vlassiouk I V (2015) Synthesis of hexagonal boron nitride monolayer: control of nucleation and crystal morphology. Chem. Mater. 27, 8041-8047.

Tan L, Han J, Mendes R G, Rümmeli M H, Liu J, Wu Q, Leng X, Zhang T, Zeng $M$, and Fu L (2015) Self-aligned single-crystalline hexagonal boron nitride arrays: toward higher integrated electronic devices. Adv. Electron. Mater. 1, 1500223.

Tay R Y, Griep M H, Mallick G, Tsang S H, Singh R S, Tumlin T, Teo E H T, and Karna S P (2014a) Growth of large single-crystalline twodimensional boron nitride hexagons on electropolished copper. Nano Lett. 14, 839-846.

Tay R Y, Li H, Tsang S H, Zhu M, Loeblein M, Jing L, Leong F N, and Teo E H T (2016a) Trimethylamine borane: A new single-source precursor for monolayer h-BN single crystals and h-BCN thin films. Chem. Mater. 28, 2180-2190.

Tay R Y, Park H J, Ryu G H, Tan D, Tsang S H, Li H, Liu W, Teo E H T, Lee Z, Lifshitz Y, and Ruoff R S (2016b) Synthesis of aligned symmetrical multifaceted monolayer hexagonal boron nitride single crystals on resolidified copper. Nanoscale 8, 2434-2444.

Tay R Y, Wang X, Tsang S H, Loh G C, Singh R S, Li H, Mallick G, and Teo E H T (2014b) A systematic study of the atmospheric pressure growth of large-area hexagonal crystalline boron nitride film. J. Mater. Chem. c 2, 1650-1657.

Wang H, Zhang X, Meng J, Yin Z, Liu X, Zhao Y, and Zhang L (2015a) Controlled growth of few-layer hexagonal boron nitride on copper foils using ion beam sputtering deposition. Small 11, 1542-1547.

Wang L, Wu B, Chen J, Liu H, Hu P, and Liu Y (2014) Monolayer hexagonal boron nitride films with large domain size and clean interface for enhancing the mobility of graphene-based field-effect transistors.
Adv. Mater. 26, 1559-1564.

Wang L, Wu B, Jiang L, Chen J, Li Y, Guo W, Hu P, and Liu Y (2015b) Growth and etching of monolayer hexagonal boron nitride. Adv. Mater. 27, 4858-4864.

Watanabe K, Taniguchi T, Niiyama T, Miya K, and Taniguchi M (2009) Far-ultraviolet plane-emission handheld device based on hexagonal boron nitride. Nat. Photon. 3, 591-594.

Wood G E, Marsden A J, Mudd J J, Walker M, Asensio M, Avila J, Chen K, Bell G R, and Wilson N R (2015) Van der Waals epitaxy of monolayer hexagonal boron nitride on copper foil: growth, crystallography and electronic band structure. 2D Mater. 2, 025003.

Wu C, Soomro A M, Sun F, Wang H, Huang Y, Wu J, Liu C, Yang X, Gao N, Chen X, Kang J, and Cai D (2016) Large-roll growth of 25-inch hexagonal BN monolayer film for self-release buffer layer of freestanding GaN wafer. Sci. Rep. 6, 34766.

Wu Q, Park J H, Park S, Jung S J, Suh H, Park N, Wongwiriyapan W, Lee S, Lee Y H, and Song Y J (2015) Single crystalline film of hexagonal boron nitride atomic monolayer by controlling nucleation seeds and domains. Sci. Rep. 5, 16159.

Yin J, Liu X, Lu W, Li J, Cao Y, Li Y, Xu Y, Li X, Zhou J, Jin C, and Guo W (2015a) Aligned growth of hexagonal boron nitride monolayer on germanium. Small 11, 5375-5380.

Yin J, Yu J, Li X, Li J, Zhou J, Zhang Z, and Guo W (2015b) Large singlecrystal hexagonal boron nitride monolayer domains with controlled morphology and straight merging boundaries. Small 11, 4497-4502.

Zhang Z, Liu Y, Yang Y, and Yakobson B I (2016) Growth mechanism and morphology of hexagonal boron nitride. Nano Lett. 16, 1398-1403.

Zhao R, Gao J, Liu Z, and Ding F (2015) The reconstructed edges of the hexagonal BN. Nanoscale 7, 9723-9730. 